\title{
Role of Paediatricians in Paediatric Ophthalmology
}

\section{Shrestha UD}

${ }^{1}$ Dr. Ujjowala Devi Shrestha, MBBS, MD, Paediatric Ophthalmology Unit, National Academy of Medical Sciences (NAMS), Tilganga Institute of Ophthalmology, Kathmandu, Nepal.

\section{Introduction}

$\mathrm{P}$ aediatricians primarily examine children. Paediatric ophthalmology is the subspecialty in ophthalmology that deals with eye diseases in children. Paediatricians can be the key personnel for early referral of the children with common avoidable causes of childhood blindness diseases to a paediatric ophthalmologist.

The common avoidable causes of childhood blindness are refractive errosr, amblyopia, ROP, VAD, xerophthalmia, ophthalmia neonatorum, congenital cataract, and retinoblastoma ${ }^{1}$. In Nepal, most of the people in the community are illiterate ${ }^{2}$. There is lack of health education and health care facilities. People are reluctant to seek medical advice for the health problems. Eye health gets the minimal importance. Children's health regarding the eyes is of a least priority.

\section{Refractive Error}

Refractive error occurs due to the unfocussed image on the retina. Myopia (nearsightedness), hyperopia (farsightedness) and astigmatism (distorted vision) are the different types of refractive errosr. Children have difficulty seeing clearly or in high hyperopia strabismus may also be present. Refractive errors are possible to correct by simple use of glasses since early childhood.

\section{Amblyopia}

Amblyopia (lazy eye) is unilateral or bilateral reduction of best-corrected visual acuity that cannot only or be directly attributed to the effect of a structural abnormality of the eye or the visual pathways. It is a diagnosis of exclusion. Amblyopia is caused by abnormal visual experience early in life resulting from strabismus, anisometropia or high bilateral refractive errors (isometropia) or stimulation visual deprivation.

\footnotetext{
Address for correspondence

Dr. Ujjowala D. Shrestha

Paediatric Ophthalmology Unit

Tilganga Institute of Ophthalmology

National Academy of Medical Sciences, NAMS

E-mail: ujjowala@gmail.com
}

\begin{abstract}
Children are primarily examined by paediatricians. In Nepal, child health is in low priority due to illiteracy, there is total lack of awareness about children's eye health. The common avoidable causes of childhood blindness are refractive errors, amblyopia, retinopathy of prematurity (ROP), vitamin $A$ deficiency (VAD), xerophthalmia, ophthalmia neonatorum $(\mathrm{ON})$, congenital cataract, and retinoblastoma. Paediatricians could be the key persons for early referral of these children to a paediatric ophthalmologist. Paediatricians can send the patients for eye examination after birth within 6 weeks, at 6 months, at one year and before going to school. In conclusion, early screening and referral by the paediatricians to the paediatric ophthalmologist prevents children from being sightless.
\end{abstract}

Key words: Paediatric ophthalmology, Refractive error, Vitamin A deficiency

With timely intervention, the reduction in visual acuity caused by amblyopia can be completely or partially reversed ${ }^{3}$. Amblyopia can be treated by glasses, patching or surgery in case of stimulation deprivation amblyopia.

\section{Antenatal care and TORCH infections}

Children's eye health care commences from the time of gestation. If mothers were exposed to prenatal TORCH infections, it could lead to strabismus, congenital cataract and macular scars ${ }^{4}$. Antenatal care is very important in maternal and child health. During the first trimester, fever and rashes can be due to Toxoplasmosis, Rubella, Cytomegalovirus, Herpes and other infections like human immunodeficiency virus (TORCH) infections. The eye health care professionals should educate the other health professionals and the community, that maternal TORCH infections can cause the eye problems like congenital cataract in the children. This information is very important for mother group.

\section{How to cite this article ?}

Shrestha UD. Role of Paediatricians in Paediatric Ophthalmology. J Nepal Paediatr Soc 2013;33(1):80-82. 


\section{Retinopathy of Prematurity (ROP)}

ROP occurs in the pre-term (born before 31 weeks of gestation) and low birth weight babies (less than 1500 grams). Early referral of these children is important to rule out ROP ${ }^{5}$. The most important factor in the pathogenesis of the ROP is prematurity and low birth weight. Other factors like sepsis, frequent blood transfusion, apnea and any problem with oxygenation are also implicated in the aetiology of ROP. Any neonate with less than 1500 grams birth weight or less than 31 weeks of gestation needs the screening. ROP causes strabismus, congenital cataract, vitreous hemorrhage and retinal detachment in children.

Paediatricians should request the paediatric ophthalmologists to examine the children's eye in the neonatology unit 6 . For babies born before 28 weeks, the first eye examination is done at 4 weeks postnatal age or at 32 weeks post-conceptional age, whichever is earlier. Babies born after 28 weeks of gestation are examined 2-3 weeks after birth.

\section{Vitamin A Deficiency (VAD)}

VAD is estimated to affect approximately one third of children under the age of five around the world? Breast milk contains vitamin A. Vitamin A prevents xerophthalmia and night blindness in children. Most of the mothers are unaware about it. This simple message from pediatrician will prevent blindness in children from VAD. Measles also causes VAD related corneal ulcer and perforation in children. Hence measles vaccination is important.

\section{Vitamin A schedule}

In areas where there is a serious problem of VAD, the first dose of vitamin $A$ is given as soon as a child with measles is seen. The dose of vitamin A supplement for children with measles, immediately on diagnosis, should be $100000 \mathrm{IU}$, by mouth, for children 6-12 months of age, and $200000 \mathrm{IU}$ for children above the age of one year. Measles is rare under the age of six months, but such young children may be given 50000 IU vitamin A. A second dose of vitamin A should be given the following day. If any of the eye signs of vitamin A deficiency are present, a third dose of vitamin A should be given 2-4 weeks later ${ }^{8}$.

\section{Ophthalmia Neonatorum (ON)}

$\mathrm{ON}$ is defined as any neonatal conjunctivitis with discharge from the eyes during the first 28 days of life, which if untreated, progresses to corneal perforation and blindness ${ }^{9}$. Its etiology may be gonococcal or nongonococcal. Paediatrician can refer the neonates with $\mathrm{ON}$ to pediatric ophthalmologist. This can prevent blindness by frequent administration of fortified topical antibiotic and single injection of ceftriaxone $50 \mathrm{mg} / \mathrm{kg}$ IM or IV for one week.

\section{Leukocoria}

Children with strabismus and visible whiteness of the pupil (cat's eye reflex), also known as leukocoria, may be due to congenital cataract or retinoblastoma ${ }^{10}$.

\section{Pediatric Cataract}

Paediatric cataract is the opacity of lens and the capsule in the paediatric age group. The management of the paediatric cataract is surgery. The surgical method is lens aspiration with or without anterior vitrectomy, and with or without intra-ocular lens implantation. The earlier the surgery better is the visual prognosis ${ }^{11}$.

\section{Retinoblastoma}

Retinoblastoma is the most common intraocular malignancy in childhood. Surgery and chemotherapy is the management for retinoblastoma. Community people are unaware about the possibility of tumors in children. They hardly know about the tumors in the eyes of children. If they are informed about it, early referral and treatment will save child's eyes and life. Initially nearly all cases were fatal. However, advances in diagnosis and treatment have resulted in a present survival rate of nearly $95 \%$. Early detection of retinoblastoma combined with improved treatment modalities has been an important factor in the reduction of morbidity and mortality. Mothers need to know that white pupillary reflex is abnormal. They should be informed that it may be due to congenital cataract or retinoblastoma ${ }^{12}$.

Table 1: Vitamin A schedule

\begin{tabular}{|c|c|c|c|}
\hline Age & Immediately on diagnosis & Next day & 2-4 weeks later (if eye signs) \\
\hline Infants less than 6 months & $50000 \mathrm{IU}$ & $50000 \mathrm{mlU}$ & $50000 \mathrm{IU}$ \\
\hline Infants aged 6-11 months & $100000 \mathrm{IU}$ & $100000 \mathrm{IU}$ & $100000 \mathrm{IU}$ \\
\hline Children aged 12 months and over & $200000 \mathrm{IU}$ & $200000 \mathrm{IU}$ & $200000 \mathrm{IU}$ \\
\hline
\end{tabular}

Note: Vitamin A capsules

$200,000 \mathrm{IU}=60,000 \mathrm{mcg}=60 \mathrm{mg}$ retinol palmitate/acetate in oily solution

$100000 \mathrm{IU}=30 \mathrm{mg}$ retinol palmitate/ acetate

$50000 \mathrm{IU}=15 \mathrm{mg}$ retinol retinol palmitate/acetate 
According to The American Academy of Ophthalmology and the American Association for Paediatric Ophthalmology and Strabismus it is essential to check children's vision. Red reflex test should be done in newborn. A second screening for eye health should be done during infancy between six months and the first birthday. For preschooler, between the ages of 3 and $3 \frac{1}{2}$, a child's vision and eye alignment should be assessed. Then finally when children go to school, paediatrician should refer the children to paediatric ophthalmologists.

\section{Conclusion}

Good visual acuity is one of the important factors for the child's physical development. Paediatric ophthalmologists can diagnose any problem of poor vision in children. If diagnosed early it can be treated promptly. Paediatricians can send the patients for eye examination after birth within 6 weeks, at 6 months, at one year and before going to school. In conclusion, early screening and referral by the paediatricians to paediatric ophthalmologist prevents one child from being blind.

\section{References}

1. Foster A. Childhood blindness. Eye 1988;2:S27S36.

2. Shrestha UD. Postoperative follow up challenge in paediatric cataract surgery in Nepal. $J$ Nepal Paediatr Soc 2011;31(3):198-201.

3. American Academy of Ophthalmology Pediatric Ophthalmology/Strabismus Panel. Preferred Practice Pattern Guidelines. Pediatric Eye Evaluations. San Francisco, CA: American Academy of Ophthalmology; 2007. Available at: http://www.aao.org/ppp. *
4. Mahalakshmi B., Therese KL, Devipriya $U$, Pushpalatha V, Margarita S., Madhavan HN. Infectious aetiology of congenital cataract based on TORCHES screening in a tertiary eye hospital in Chennai, Tamil Nadu, India. Indian J Med Res 2010;131(4):559-564.

5. Shah VA, Yeo CL, Ling Y, Ho LY. Incidence, Risk Factors of Retinopathy of Prematurity Among Very Low Birth Weight Infants in Singapore. Annals Acad Med 2005;34(2):169-178.

6. Aggarwal R, Agarwal R, Deorari AK, Paul VK. Retinopathy of prematurity. Indian $J$ Pediatr 2002;69(1):83-6.

7. Global prevalence of vitamin A deficiency in populations at risk 1995-2005. WHO global database on vitamin A deficiency. World Health Org 2009

8. The child, measles and the eye. World Health Org 1993 [updated 2004].

9. Vedantham V. Prophylaxis of ophthalmia neonatorum. Br J Ophthalmol 2004;88(10):1352.

10. Balmer A., Munier F. Differential diagnosis of leukocoria and strabismus, firs presenting signs of retinoblastoma. Clin Ophthalmol 2007;1(4):431439.

11. Shrestha UD, Shrestha MK. Clinical Profile of Paediatric Cataract and Surgery in a Tertiary Eye Care Centre. J Nepal Paediatr Soc 2012; 32(1) 1418.

12. Metz Thomas H., Nelson Leonard B, Shields Carol L. Pupillary Dilation by Pediatricians. Pediatrics 1999;104(4):958-60. 